\title{
Uptake and Dissolution of Gaseous Ethanol in Sulfuric Acid
}

Rebecca R. Michelsen, Sarah J.R. Staton, and Laura T. Iraci

\section{Supporting Information}

Table S1. Measured Effective Henry's Law Coefficients for Uptake of Ethanol into Aqueous Sulfuric Acid Solutions with T, D and $H^{*}$ Corrected for Maximum Possible Thermal Transpiration Effect

\begin{tabular}{|c|c|c|c|c|}
\hline $\begin{array}{l}\mathrm{H}_{2} \mathrm{SO}_{4} / \mathrm{H}_{2} \mathrm{O} \\
\text { composition } \\
\end{array}$ & $\begin{array}{c}T \\
(\mathrm{~K}) \\
\end{array}$ & $\begin{array}{c}H * \sqrt{D} \\
\left(\mathrm{M} \mathrm{cm} \mathrm{atm}^{-1} \mathrm{~s}^{-1 / 2}\right) \\
\end{array}$ & $\begin{array}{c}D \\
\left(\mathrm{~cm}^{2} \mathrm{~s}^{-1}\right) \\
\end{array}$ & $\begin{array}{c}H^{*} \\
\left(\mathrm{M} \mathrm{atm}^{-1}\right) \\
\end{array}$ \\
\hline \multirow{10}{*}{$66.3 \mathrm{wt} \% \mathrm{H}_{2} \mathrm{SO}_{4}$} & 213.3 & $4.18 \times 10^{3}$ & $1.27 \times 10^{-8}$ & $3.7 \times 10^{7}$ \\
\hline & 217.8 & $2.47 \times 10^{3}$ & $2.25 \times 10^{-8}$ & $1.7 \times 10^{7}$ \\
\hline & 219.7 & $2.33 \times 10^{3}$ & $2.80 \times 10^{-8}$ & $1.4 \times 10^{7}$ \\
\hline & 224.6 & $1.49 \times 10^{3}$ & $4.69 \times 10^{-8}$ & $6.9 \times 10^{6}$ \\
\hline & 224.7 & $1.19 \times 10^{3}$ & $4.73 \times 10^{-8}$ & $5.5 \times 10^{6}$ \\
\hline & 228.8 & $1.02 \times 10^{3}$ & $6.92 \times 10^{-8}$ & $3.9 \times 10^{6}$ \\
\hline & 228.9 & $9.05 \times 10^{2}$ & $6.98 \times 10^{-8}$ & $3.4 \times 10^{6}$ \\
\hline & 233.0 & $6.71 \times 10^{2}$ & $9.87 \times 10^{-7}$ & $2.1 \times 10^{6}$ \\
\hline & 235.7 & $2.45 \times 10^{2}$ & $1.21 \times 10^{-7}$ & $7.1 \times 10^{5}$ \\
\hline & 235.8 & $3.29 \times 10^{2}$ & $1.22 \times 10^{-7}$ & $9.4 \times 10^{5}$ \\
\hline \multirow[t]{10}{*}{$59.1 \mathrm{wt} \% \mathrm{H}_{2} \mathrm{SO}_{4}$} & 207.5 & $1.17 \times 10^{3}$ & $1.64 \times 10^{-8}$ & $9.2 \times 10^{6}$ \\
\hline & 217.4 & $1.08 \times 10^{3}$ & $5.12 \times 10^{-8}$ & $4.8 \times 10^{6}$ \\
\hline & 217.5 & $9.94 \times 10^{2}$ & $5.17 \times 10^{-8}$ & $4.4 \times 10^{6}$ \\
\hline & 217.6 & $7.68 \times 10^{2}$ & $5.22 \times 10^{-8}$ & $3.4 \times 10^{6}$ \\
\hline & 222.0 & $3.48 \times 10^{2}$ & $7.90 \times 10^{-8}$ & $1.2 \times 10^{6}$ \\
\hline & 227.9 & $2.28 \times 10^{2}$ & $1.29 \times 10^{-7}$ & $6.3 \times 10^{5}$ \\
\hline & 229.4 & $2.20 \times 10^{2}$ & $1.45 \times 10^{-7}$ & $5.8 \times 10^{5}$ \\
\hline & 229.5 & $2.18 \times 10^{2}$ & $1.46 \times 10^{-7}$ & $5.7 \times 10^{5}$ \\
\hline & 235.0 & $1.03 \times 10^{2}$ & $2.16 \times 10^{-7}$ & $2.2 \times 10^{5}$ \\
\hline & 236.3 & $9.57 \times 10^{1}$ & $2.35 \times 10^{-7}$ & $2.0 \times 10^{5}$ \\
\hline \multirow[t]{12}{*}{$38.5 \mathrm{wt} \% \mathrm{H}_{2} \mathrm{SO}_{4}$} & 207.5 & $3.82 \times 10^{2}$ & $5.55 \times 10^{-8}$ & $1.6 \times 10^{6}$ \\
\hline & 212.2 & $1.22 \times 10^{2}$ & $9.37 \times 10^{-8}$ & $4.0 \times 10^{5}$ \\
\hline & 212.5 & $2.07 \times 10^{2}$ & $9.64 \times 10^{-8}$ & $6.7 \times 10^{5}$ \\
\hline & 217.6 & $1.24 \times 10^{2}$ & $1.58 \times 10^{-7}$ & $3.1 \times 10^{5}$ \\
\hline & 217.6 & $1.76 \times 10^{2}$ & $1.58 \times 10^{-7}$ & $4.4 \times 10^{5}$ \\
\hline & 218.2 & $1.25 \times 10^{2}$ & $1.66 \times 10^{-7}$ & $3.1 \times 10^{5}$ \\
\hline & 223.4 & $3.49 \times 10^{1}$ & $2.58 \times 10^{-7}$ & $6.9 \times 10^{4}$ \\
\hline & 223.6 & $3.49 \times 10^{1}$ & $2.62 \times 10^{-7}$ & $6.8 \times 10^{4}$ \\
\hline & 226.0 & $2.93 \times 10^{1}$ & $3.14 \times 10^{-7}$ & $5.2 \times 10^{4}$ \\
\hline & 226.1 & $2.07 \times 10^{1}$ & $3.17 \times 10^{-7}$ & $3.7 \times 10^{4}$ \\
\hline & 226.4 & $3.47 \times 10^{1}$ & $3.24 \times 10^{-7}$ & $6.1 \times 10^{4}$ \\
\hline & 226.9 & $3.42 \times 10^{1}$ & $3.36 \times 10^{-7}$ & $5.9 \times 10^{4}$ \\
\hline
\end{tabular}


Table S2. Thermodynamic Parameters for Ethanol Dissolution in Aqueous Sulfuric Acid from Measurements Corrected for Maximum Possible Thermal Transpiration Effect

\begin{tabular}{ccccc}
\hline $\begin{array}{c}\mathrm{H}_{2} \mathrm{SO}_{4} / \mathrm{H}_{2} \mathrm{O} \\
\text { composition }\end{array}$ & $X_{\mathrm{H}_{2} \mathrm{SO}_{4}}$ & $\begin{array}{c}M_{\text {solv }}{ }^{a} \\
\left(\mathrm{~mol} \mathrm{~L}^{-1}\right)\end{array}$ & $\begin{array}{c}\Delta H^{\circ} \\
\left(\mathrm{kJ} \mathrm{mol}^{-1}\right)\end{array}$ & $\begin{array}{c}\Delta S^{\circ} \\
\left(\mathrm{J} \mathrm{mol}^{-1} \mathrm{~K}^{-1}\right)\end{array}$ \\
\hline $0 \mathrm{wt} \% \mathrm{H}_{2} \mathrm{SO}_{4}$ & 0.00 & 55.6 & -54.8 & -174 \\
$38.5 \mathrm{wt} \% \mathrm{H}_{2} \mathrm{SO}_{4}$ & 0.10 & 46.0 & -69.5 & -248 \\
$59.1 \mathrm{wt} \% \mathrm{H}_{2} \mathrm{SO}_{4}$ & 0.21 & 35.3 & -58.7 & -175 \\
$66.3 \mathrm{wt} \% \mathrm{H}_{2} \mathrm{SO}_{4}$ & 0.27 & 30.7 & -68.1 & -202 \\
\hline $38.5 \mathrm{wt} \% \mathrm{H}_{2} \mathrm{SO}_{4}$ & 0.10 & 46.0 & -53.2 & -174 \\
$59.1 \mathrm{wt} \% \mathrm{H}_{2} \mathrm{SO}_{4}$ & 0.21 & 35.3 & -58.3 & -174 \\
$66.3 \mathrm{wt} \% \mathrm{H}_{2} \mathrm{SO}_{4}$ & 0.27 & 30.7 & -61.8 & -174 \\
\hline
\end{tabular}

${ }^{a}$ Calculated at $220 \mathrm{~K}$.

In order to determine the solubility of ethanol using Equation S1 at conditions other than those studied, and including the correction for thermal transpiration, $A$ can be calculated from Equation S2 with $\Delta S^{\circ}=-174 \mathrm{~J} \mathrm{~mol}^{-1} \mathrm{~K}^{-1}$, and $B$ can be calculated from the empirical relation Equation S3 where $x_{\mathrm{H}_{2} \mathrm{SO}_{4}}$ is the mole fraction of sulfuric acid in the solution.

$$
\begin{aligned}
& \log H^{*}=A+1000 B / T \\
& A=\Delta S^{\circ} / 2.303 R+\log M_{\text {solv }} \\
& B=4.535 *\left(x_{\mathrm{H}_{2} \mathrm{SO}_{4}}\right)^{2}+1.095 *\left(x_{\mathrm{H}_{2} \mathrm{SO}_{4}}\right)+2.617
\end{aligned}
$$

Table S3. Measured Effective Henry's Law Coefficients for Equilibrium Vapor Pressure of Ethanol over Mixed Solutions with $T, P_{\mathrm{EtOH}}$, and $H^{*}$ Corrected for Maximum Possible Thermal Transpiration Effect

\begin{tabular}{ccccc}
\hline $\begin{array}{c}\text { initial } \mathrm{H}_{2} \mathrm{SO}_{4} / \mathrm{H}_{2} \mathrm{O} \\
\text { composition }\end{array}$ & $\begin{array}{c}{[\mathrm{EtOH}]} \\
(\mathrm{M})\end{array}$ & $\begin{array}{c}T \\
(\mathrm{~K})\end{array}$ & $\begin{array}{c}P_{\text {EtOH }} \\
(\mathrm{atm})\end{array}$ & $\begin{array}{c}H^{*} \\
\left(\mathrm{M} \mathrm{atm}^{-1}\right)\end{array}$ \\
\hline $59.1 \mathrm{wt} \% \mathrm{H}_{2} \mathrm{SO}_{4}$ & 0.5 & 213.6 & $1.1 \times 10^{-7}$ & $4.7 \times 10^{6}$ \\
& & 223.3 & $3.9 \times 10^{-7}$ & $1.3 \times 10^{6}$ \\
& & 230.2 & $1.0 \times 10^{-6}$ & $4.9 \times 10^{5}$ \\
\hline $38.5 \mathrm{wt} \% \mathrm{H}_{2} \mathrm{SO}_{4}$ & 0.1 & 212.8 & $2.5 \times 10^{-7}$ & $3.9 \times 10^{5}$ \\
& & 218.1 & $5.0 \times 10^{-7}$ & $2.0 \times 10^{5}$ \\
& & 222.7 & $9.5 \times 10^{-7}$ & $1.0 \times 10^{5}$ \\
\hline
\end{tabular}

